\title{
Surgical treatment of MRONJ in oncology and non-oncology patients: a retrospective study
}

\author{
Paolo Garzino Demo ${ }^{1}$ \\ 1 University of Turin \\ Funding: The author(s) received no specific funding for this work. \\ Potential competing interests: The author(s) declared that no potential competing interests exist.
}

\section{Abstract}

Medication-related osteonecrosis of the jaw (MRONJ) is a severe side effect caused by antiresorptive antiangiogenic medication for different pathologies. Although the main factors that seem to increase the risk of ONJ have been identified and the main scientific societies have recently disclosed rules of good behaviour regarding prevention, diagnosis and patient management, there are still no guidelines on therapeutic strategies to be adopted. In general, if conservative treatment fails, surgical treatment is considered, including local debridement, osteoplasty, and marginal or segmental osteotomy. To date, in literature, cohorts of heterogeneous patients with MRONJ have been analysed for a long time, with a lack of uniformity in information and difficulties in interpreting data. This retrospective study evaluates the results of surgical treatment of 62 patients with stage II-III of MRONJ according to the American Association of Oral and Maxillofacial Surgeons criteria, evaluated at the Department of Maxillofacial Surgery, in the University of Turin (Italy), by subdividing patients into different cohorts firstly according to the primary pathology, i,e. oncological and nononcological, and secondly, according to the drug or combination of drugs taken.

Introduction: MRONJs are adverse events secondary to drugs taken to treat various oncological and non-oncological diseases. The clinical and radiological characteristics of MRONs depend on the type of causative drug, the time of administration and its dosage. Assessment of therapeutic success and cure rates may vary depending on whether the patient is oncological or non-oncological.

Aims: the first aim was to evaluate the treatment results for stage II-III MRONJ, irrespective of the underlying pathology. The second objective was to evaluate the results by dividing the clinical case series into two cohorts of patients divided according to the underlying pathology, i.e. oncological and non-oncological.

Materials and methods: the surgery results in 64 sites of MRONJ operated in 62 patients stage II-III of MRONJ patients were studied. The patients were divided into different cohorts according to the type of drug is taken and the underlying pathology: 31 oncological and 31 non-oncological patients. The results of surgical treatment in the two cohorts were compared.

Statistical analysis: descriptive statistics were generated for the main quantitative variables; survival curves were calculated using the Kaplan-Meier algorithm. A log-rank test was used. The difference in survival between subgroups was 
tested using the log-rank statistic.

Results: considering the surgery results by dividing the treated patients into oncological and non-oncological patients: oncological patients had a cure rate of $93 \%$, while non-oncological patients had a cure rate of $59.4 \%$. $(p<0.05)$

Conclusions: The MRONJ therapy results vary depending on the drug taken, the reason for taking it and the route of administration. 